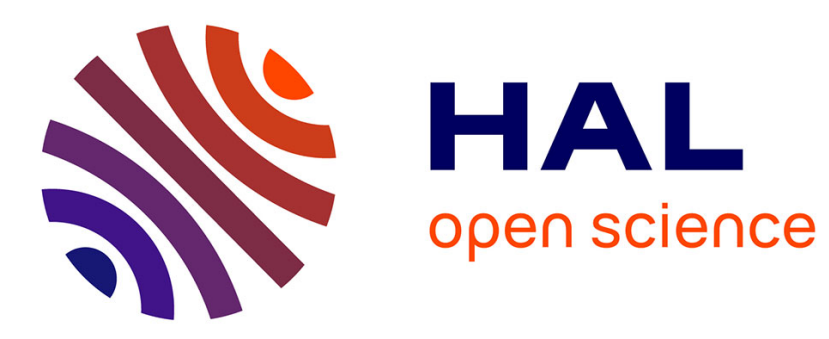

\title{
Role of Solvent in the Energy Level Alignment of Dye-Sensitized NiO Interfaces
}

Simone Piccinin, Dario Rocca, Mariachiara Pastore

\section{To cite this version:}

Simone Piccinin, Dario Rocca, Mariachiara Pastore. Role of Solvent in the Energy Level Alignment of Dye-Sensitized NiO Interfaces. Journal of Physical Chemistry C, 2017, 121 (40), pp.22286-22294. 10.1021/acs.jpcc.7b08463 . hal-02387081

\section{HAL Id: hal-02387081 \\ https://hal.science/hal-02387081}

Submitted on 10 Nov 2021

HAL is a multi-disciplinary open access archive for the deposit and dissemination of scientific research documents, whether they are published or not. The documents may come from teaching and research institutions in France or abroad, or from public or private research centers.
L'archive ouverte pluridisciplinaire HAL, est destinée au dépôt et à la diffusion de documents scientifiques de niveau recherche, publiés ou non, émanant des établissements d'enseignement et de recherche français ou étrangers, des laboratoires publics ou privés. 


\title{
The Role of Solvent in the Energy Level Alignment of Dye-sensitized NiO Interfaces
}

\author{
Simone Piccinin, ${ }^{*, \dagger}$ Dario Rocca, ${ }^{\ddagger}, \mathbb{I}$ and Mariachiara Pastore ${ }^{\S, \|}$ \\ CNR-IOM DEMOCRITOS c/o SISSA, Via Bonomea 265, 34136 Trieste, Italy, Université de \\ Lorraine, CRMㄴ, UMR 7036, 54506 Vandoeuvre-lès-Nancy, France, CNRS, CRM², UMR 7036, \\ 54506 Vandoeuvre-lès-Nancy, France, CNRS, SRSMC, UMR 7565, Boulevard des Aiguillettes, BP \\ 7023954506 Vandoeuvre-les-Nancy Cedex, France, and Université de Lorraine, SRSMC, UMR \\ 7565, Boulevard des Aiguillettes, BP 7023954506 Vandoeuvre-les-Nancy Cedex, France \\ E-mail: piccinin@iom.cnr.it
}

\begin{abstract}
p-type dye-sensitized solar cells (DSCs), based on hole injection from the photoexcited dye into the valence band of a semiconductor like $\mathrm{NiO}$, represent an attractive device to exploit solar radiation. However, they perform rather poorly compared to $n$-type DSCs, for reasons that are currently heavily debated. In this work we use simulations based on density functional theory and first principles molecular dynamics to investigate the interface between $\mathrm{NiO}$ and C343, a widely used dye. We focus on the structure of the interface and on the energy level alignment. Accounting explicitly for the role of the solvent, we find that the $\mathrm{NiO}$ surface binds
\end{abstract}

\footnotetext{
CNR-IOM DEMOCRITOS c/o SISSA, Via Bonomea 265, 34136 Trieste, Italy

ثniversité de Lorraine, CRM², UMR 7036, 54506 Vandoeuvre-lès-Nancy, France

${ }^{\mathbb{I}} \mathrm{CNRS}, \mathrm{CRM}^{2}$, UMR 7036, 54506 Vandoeuvre-lès-Nancy, France

${ }^{\S}$ CNRS, SRSMC, UMR 7565, Boulevard des Aiguillettes, BP 7023954506 Vandoeuvre-les-Nancy Cedex, France

"Université de Lorraine, SRSMC, UMR 7565, Boulevard des Aiguillettes, BP 7023954506 Vandoeuvre-les-Nancy Cedex, France
} 
a monolayer of water molecules that, through the orientation of their dipoles, align favorably the dye's HOMO relative to NiO's valence band. We also show that an alignment consistent with the experimental evidence of ultrafast injection can only be obtained accounting explicitly for the role of the solvent.

\section{Introduction}

In the context of solar energy exploitation, dye-sensitized solar cells (DSCs) ${ }^{1-3}$ and dye-sensitized photoelectrosynthetic cells (DSPECs) ${ }^{4-7}$ offer the promise of cost effective sunlight conversion and storage, respectively. Since the seminal paper by O'Regan and Grätzel in $1991,{ }^{8}$ hybrid/organic photovoltaic devices have attracted significant research interest, with the recent launch of the first commercial product. The heart of a $n$-type DSC is the photoanode, where a mesoporous oxide layer, usually $\mathrm{TiO}_{2}$ or $\mathrm{ZnO}$, is sensitized by a monolayer of dyes, chemically bound to the semiconductor nanoparticles. Upon solar irradiation, the adsorbed sensitizers are able to inject the photoexcited electrons in the manifold of the conduction band (CB) states of the semiconductor, typically at the femtosecond time scale. Injected electrons travel through the mesoporous film and are collected by the conductive layer of the photoanode electrode, while the oxidized dye is rapidly reduced by the electron mediator donor in solution or by the hole transporting material (HTM) in solid-state devices. The collected electrons flow in the external circuit, producing a photocurrent, and reach the counter-electrode, where the circuit is closed by the reduction of the electron mediator acceptor. Alternatively, in a $p$-type DSC, the photoactive electrode is a dye-functionalized nanostructured wide band gap $p$-type semiconductor (usually $\mathrm{NiO}$ ). ${ }^{9-12} \mathrm{In}$ this case the functioning mechanism is inverse, with hole injection from the photoexcited dye to the $p$-type semiconductor valence band (VB) and the concomitant electron transfer to the redox mediator and final electron collection at the anode (Fig. Figure 1).

This reverse architecture can be either combined with the $n$-type DSC in a tandem device, in such a way to surpass the theoretical upper limit of $30 \%$ efficiency of a single photoactive electode cell, ${ }^{13-16}$ or employed in a DSPEC at the photocathode for water reduction. ${ }^{17}$ The latter is di- 


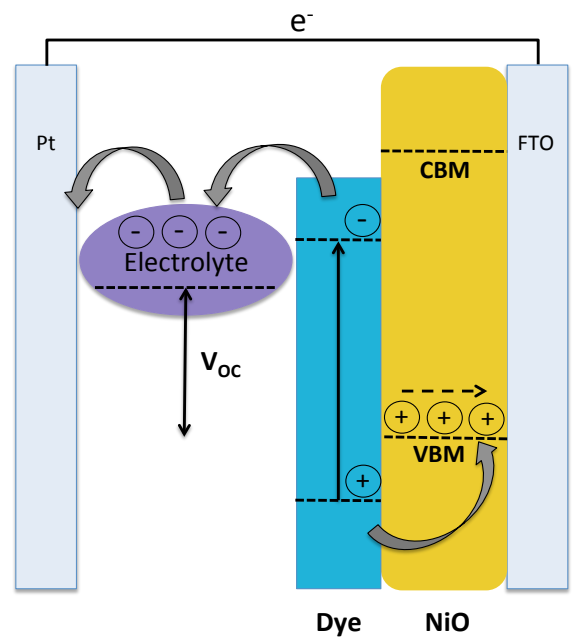

Figure 1: Operational mechanism of a p-type DSC

rectly inspired by a DSC, where molecular systems (dyes and catalyst) are adsorbed on the surface of a wide band-gap semiconductor (typically $\mathrm{TiO}_{2}$ at the photoanode and $\mathrm{NiO}$ at the photocathode). Dye-sensitized photoanode and photocathodes can be then combined in a tandem cell for water splitting. Upon solar light absorption, at the photoanode, the dye's photo-excited electrons are injected into the semiconductor $\mathrm{CB}$, while the concomitant holes are transferred to the catalyst for water oxidation. The injected electrons are then collected at the photocathode, where, upon dye's excitation, the electrons are transferred to the water reduction catalyst and the holes to the p-type semiconductor VB. In such a photoelectrosynthetic device, oxygen is produced at the dye-sensitized semiconductor photoanode, while hydrogen is produced at the photocathode. ${ }^{18-20}$ Despite the huge interest in developing efficient $p$-type electrodes, their solar-energy-to-electricenergy conversion efficiency is still one order of magnitude smaller that the $n$-type DSCs. ${ }^{21,22}$ Regardless of the efforts in materials optimization (redox mediator, ${ }^{21-23}$ dye structure, ${ }^{14,24-26}$ use of additives ${ }^{27}$ and semiconductor doping strategies ${ }^{28}$ ), the measured photocurrent densities are still extremely low when compared to those obtained for $\mathrm{TiO}_{2}$ based photoanodes, leading to the common assumption that nanostructured $\mathrm{NiO}$, characterized by a relatively low bulk hole conductivity, ${ }^{29}$ is intrisically not optimal for DSCs applications. ${ }^{29}$ Since 2005, when the ultrafast (ca. 200 fs) photoinduced electron transfer from nanostructured $\mathrm{NiO}$ to the cumarin 343 (C343) sensitizer 
was reported for the first time, ${ }^{30}$ a number of Transient Absorption Spectroscopy (TAS) investigations have been carried out for dye-sensitized NiO electrodes. ${ }^{20,25,31-34}$ All these experiments have shown for the best dye architectures an ultrafast dynamics for the hole injection into the semiconductor (oxidized dye reduction) and a remarkably fast back recombination reactions (ps timescale). The reason for these poor photocurrents and device efficiencies has, therefore, to be found in fast back recombination pathways at the solid/liquid interfaces (NiO/dye and NiO/electrolyte) ${ }^{28,35}$ rather than in a poor intrinsic hole conductivity of mesoporous $\mathrm{NiO} .{ }^{11,36}$ Indeed, as discussed by Hammarström and co-workers ${ }^{28}$ for a bare mesoporous $\mathrm{NiO}$ film, the electron transfer from the redox couple $\left(\mathrm{I}_{3}^{-} / \mathrm{I}^{-}\right)$to the metal oxide takes place already at low potentials, assisted by both a favorable redox potentials alignment between $\mathrm{NiO}$ and the electrolyte reductive species ${ }^{23,37}$ and the presence of trap states above the semiconductor VB edge. Further evidence of the crucial role of the electrolyte/ $\mathrm{NiO}$ interaction has also recently been reported in an X-ray Photoelectron Spectroscopy (XPS) study for the acetonitrile solvated NiO interface, where the adsorption of $\mathrm{I}_{3}^{-}$ and $\mathrm{I}^{-}$at the $\mathrm{NiO}$ mesoporous surface is monitored at different applied potentials. Also in this experiment the dye monolayer, with its potential surface passivation and bands shift effects, was not present at the $\mathrm{NiO} /$ solvent interface. As a matter of fact, the characterization of the electronic and structural properties of dye-sensitized $\mathrm{NiO}$ interfaces in both $p$-type DSCs and water reduction photocathodes is still poor when compared to the level of understanding reached for $\mathrm{TiO}_{2}$ sensitized photoanodes. The same considerations apply to theoretical studies that, while having played a decisive role assisting experimental research in the development of the $\mathrm{TiO}_{2}$ based DSCs ${ }^{38-41,41-46}$ and perovskites solar cells, ${ }^{47,48}$ are still lacking in the modeling of the dye/NiO heterointerface. The first theoretical work on a dye-sensitized $\mathrm{NiO}$ interface model has, in fact, appeared only very recently: ${ }^{49}$ The cumarin 343 dye anchored by both phosphonic and carboxylic acid functionalities on the $\mathrm{NiO}(100)$ surface was investigated by using periodic spin polarized $\mathrm{DFT}+\mathrm{U}$ calculations in gas phase. Other two studies employing hybrid functionals and localized basis sets have been reported later, considering the adsorption of a perylene monoimide dye ${ }^{50}$ and the screening of different anchoring groups at the dye functionalized $\mathrm{NiO}$ surface. ${ }^{51}$ In all these studies, the energy 
level alignment across the interface, and in particular the relative position of the dye's HOMO with respect to $\mathrm{NiO}$ 's $\mathrm{VB}$, is found to be extremely sensitive to the anchoring group (carboxylic acid, phosphonic acid, alkoxilane) and binding mode (mododentate, bidentate, tridentate) of the dye. Indeed, the reduction of the interfacial electric dipole significantly increases the energy difference between the metal oxide VB and the dye's HOMO, therefore increasing the driving force for the hole injection process. ${ }^{49}$ Due to the high sensitivity of the dye/surface level alignment on the changes of the interfacial electrostatic potential, limiting the calculations to the equilibrium geometry in gas phase (i.e. neglecting thermal fluctuations and the effect of the solvent) can only provide a rough approximation to the system energetics.

In an effort to improve the predictive power of theoretical simulations for these systems, in this work we tackle the dynamical modeling of the full interface, including the water environment. By using the DFT $+\mathrm{U}$ approach and including explicit water molecules we perform first-principles molecular dynamics simulations of the $\mathrm{NiO}(100)$ surface sensitized by the $\mathrm{C} 343$ dye anchored via a phosphonic acid. We then investigate the electronic structure of the system and the level alignment across the interface at selected snapshots extracted from the dynamics. This analysis is carried out by using both DFT+U and hybrid functionals (HSE06) and by comparing the results obtained with explicit water to those obtained using an implicit solvent model. We find that the use of an explicit solvation for the dye/oxide interface model is essential to have a reliable band offset energetics along the MD trajectory. Indeed, the formation of a fairly rigid solvent layer bound to the surface causes a sizable electrostatic effect and induces a change in the dye binding mode after few picoseconds of dynamics. DFT+U and hybrid functionals, on the other hand, give a very similar level alignment across the interface. 


\section{Theoretical Methods}

To investigate how $\mathrm{C} 343$ binds to $\mathrm{NiO}(100)$ we employ DFT calculations with the PBE exchange and correlation functional, ${ }^{52}$ adding a Hubbard $\mathrm{U}$ term to Ni(d) electrons. Following Cococcioni and de Gironcoli, ${ }^{53}$ we adopted the value $\mathrm{U}=4.6 \mathrm{eV}$ derived from linear response. For static calculations, we use ultrasoft pseudopotentials ${ }^{54}$ and a plane waves basis set, with a cutoff of 30 Ry for the wave functions and 300 Ry for the charge density. Van der Waals interactions are modeled using Grimme's method. ${ }^{55}$ These calculations have been performed with the Quantum ESPRESSO software. ${ }^{56}$ We adopt a slab geometry for $\mathrm{NiO}(100)$, consisting of 6 layers in a $(2 \times 2)$ unit cell, periodically repeated in the three dimensions. The dye is adsorbed on one side only, and to remove the spurious dipolar interaction among different replicas of the system in the direction perpendicular to the slab we introduce a compensating dipole in the vacuum region. The vacuum region is about $18 \AA$. The Brillouin zone is sampled with a uniform $4 \times 4 \times 1$ grid of K-points centered at Gamma. The projections of the Kohn-Sham states of the full systems on the HOMO of the isolated dye have been performed with the molecularpdos.x tool in Quantum ESPRESSO. ${ }^{57}$

Molecular dynamics simulations $(\sim 33 \mathrm{ps})$ in explicit water have been performed with the CP2K code, using GTH norm-conserving pseudopotentials, MOLOPT-DZVP basis set, 500 Ry cutoff for the charge density, and sampling the Brillouin zone at the Gamma point. Also in this case we employed the PBE+U approach with Grimme's van der Waals corrections. The temperature is set to $360 \mathrm{~K}$ through the use of the canonical sampling velocity rescaling thermostat. ${ }^{58}$ The surface unit cell adopted is a $(2 \times 2)$, with the simulation box having dimensions $8.35 \times 8.35 \times$ $33.40 \AA^{3}$, and the solvent consists in 39 water molecules. The time step is set to $1 \mathrm{fs}$ and we use deuterium mass for hydrogen atoms. The first 3 ps of the dynamics have been used for equilibration and have been discarded. From the $\sim 30$ ps production run we extracted a sequence of timeequispaced snapshots $(0.5 \mathrm{ps})$ that have been analyzed with single-point $\mathrm{PBE}+\mathrm{U}$ calculations with Quantum ESPRESSO and with the HSE06 hybrid functional ${ }^{59}$ using CP2K. In HSE06 calculations we reduce the fraction of exact exchange from the original $25 \%$ to $20 \%$ in order to obtain a gap for $\mathrm{NiO}$ compatible with experimental measurements. 


\section{Results and Discussion}

\section{Isolated and adsorption systems}

For $\mathrm{NiO}$ bulk we considered the experimental rocksalt structure with a lattice constant of 4.176 $\AA$. The spins of Ni atoms are ferromagnetically aligned within the (111) planes, and are antiferromagnetically ordered across different (111) planes, resulting in an AF2 phase. The experimental optical absorption spectrum of $\mathrm{NiO}$ shows an onset of absorption at $3.1 \mathrm{eV}$, reaches its maximum at $4.3 \mathrm{eV},{ }^{60}$ and the optical gap is usually extrapolated to $4.0 \mathrm{eV} .{ }^{61}$ The fundamental gap measured through direct and inverse photoemission spectroscopy is in the 3.2-5.7 eV range, ${ }^{61,62}$ depending on how the value is extracted from the measured spectra, while is $4.0 \mathrm{eV}$ from $\mathrm{X}$-ray emission and absorption spectroscopy. ${ }^{63}$

There is a fairly large number of theoretical works on the electronic structure of bulk $\mathrm{NiO}$ present in the literature, using DFT methods (GGA functionals, DFT+U, hybrid functionals) as well as many-body perturbation theory $(\mathrm{GW}) .{ }^{64-67}$ The electronic structure of $\mathrm{NiO}$, and its band gap in particular, depends rather strongly of the choice of the method. Using the PBE+U approach with a value of $\mathrm{U}$ for $\mathrm{Ni}(\mathrm{d})$ states of $4.6 \mathrm{eV}$ as obtained from linear response, ${ }^{53}$ the computed direct gap we obtain with our setup is $3.07 \mathrm{eV}$ (indirect gap $2.53 \mathrm{eV}$ ). The top of the valence band has a mixed $\mathrm{O}(\mathrm{p})-\mathrm{Ni}(\mathrm{d})$ character, while the bottom of the conduction band is dominated by $\mathrm{Ni}(\mathrm{d})$ levels (see Fig. S2 in Supporting Information (SI)), in agreement with previous investigations. ${ }^{53,68-70}$ Hybrid functional calculations lead to a larger gap, $4.95 \mathrm{eV}$ using HSE06 and 5.76 eV using PBE0, both of which include a fraction $X=25 \%$ of exact exchange. Lowering $X$ to $20 \%$ in HSE06 leads to a gap of $4.31 \mathrm{eV}$, in better agreement with the experimental value. All calculations with the HSE06 functional have therefore been performed with $\mathrm{X}=20 \%$. Apart from the expected effect on the gap, ${ }^{51,71}$ the inclusion of exact exchange modifies the character of the top of the valence band: while it is predominantly $\mathrm{O}(\mathrm{p})$ in the case of $\mathrm{PBE}+\mathrm{U}$, it is mostly $\mathrm{Ni}(\mathrm{d})$ in the case of HSE06, as shown by the projected density of states (PDOS) in Fig. S2.

As dye sensitizer we investigate a derivative of C343, because of its well characterized inter- 
action with the $\mathrm{NiO}$ surface. ${ }^{19,30,49,72-75}$ The C343 dye considered in our work features a terminal phosphonic acid $\left(-\mathrm{PO}(\mathrm{OH})_{2}\right)$ anchoring group, which guarantees a superior grafting stability (stronger adsorption) $49,76,77$ in water environment, where the carboxylate group, normally employed in DSCs, undergoes desorption from the oxide surface. ${ }^{78,79}$ We examined two possible conformers: ${ }^{80,81}$ one where one of the $\mathrm{H}$ atoms of the phosphonate is $\mathrm{H}$-bonded to the oxygen of the carbonyl group of the coumarin (H-up), and another where both $\mathrm{H}$ atoms of the phosphonate point away from it (H-down) (see Fig. S3). In gas phase, the H-bonded isomer is slightly favored, by $0.20 \mathrm{eV}$ using the PBE functional.

To model the surface of $\mathrm{NiO}$ we considered the (100) facet, since this is the most stable termination. Geometry optimization of the system were performed with a 6-layer slab of $\mathrm{NiO}(100)$, using a $(2 \times 2)$ surface unit cell, obtaining very small relaxation $(\sim 1 \%$ expansion of the first two layers) with respect to ideal bulk positions. Using the Kohn-Sham eigenvalues (relative to vacuum) as estimates of electron removal energies, $\mathrm{PBE}+\mathrm{U}$ calculations place the valence band maximum $(\mathrm{VBM})$ of $\mathrm{NiO}$ at $-5.27 \mathrm{eV}$, while a value of $-5.70 \mathrm{eV}$ is obtained at HSE06 level of theory (see data in Tab. S1 in SI). The experimental values for the VBM of $\mathrm{NiO}$ (relative to vacuum) present in the literature vary considerably: from $-4.8 \mathrm{eV}$ measured through photoemission spectroscopy, ${ }^{82}$ to $-5.0 \mathrm{eV}$ obtained through electrochemical measurements, ${ }^{9,83}$ to $-5.8 \mathrm{eV}$ obtained from thermionic emission, ${ }^{84}$ with the most frequently reported estimate being $-5.0 \mathrm{eV} .{ }^{85}$

The HOMO of the $\mathrm{C} 343-\mathrm{PO}(\mathrm{OH})_{2}$ molecule is calculated at $-5.25 \mathrm{eV}$ and $-5.63 \mathrm{eV}$ at $\mathrm{PBE}$ and HSE06 level of theory, respectively, thus in both cases slightly above (within $0.1 \mathrm{eV}$ ) the NiO's VBM. The experimental ground state oxidation potential (GSOP) is available only for the C343$\mathrm{COOH}$ dye in Dimethylformamide (DMF) solution, and is measured at $-1.2 \mathrm{~V}$ vs NHE (ca. 5.65 $\mathrm{eV}$ with respect to the vacuum), from which a value of $-5.9 \mathrm{eV}$ is estimated for the position of the HOMO relative to vacuum. ${ }^{86}$ Our estimate for the HOMO of $\mathrm{C} 343-\mathrm{COOH}$ in DMF is -4.90 $\mathrm{eV}$ for PBE and -5.28 eV for HSE06 (i.e. a shift of $+0.35 \mathrm{eV}$ with respect to $\mathrm{C} 343-\mathrm{PO}(\mathrm{OH})_{2}$ in vacuum, see SI for details). There is therefore a fairly large discrepancy with the experimental value of the dye's HOMO. While this comparison refers to isolated systems and the focus of 
this work is mainly on the level alignment across the interface in solution, we need to keep in mind that the dye's HOMO could be further stabilized by more accurate approximations. Many body perturbation theory (i.e. GW calculations), in particular, could lead to better agreement with experiment compared to our estimates obtained from the Kohn-Sham eigenvalues. ${ }^{87,88}$ However, when considering the full interface these calculations are computationally very demanding and are currently the subject of ongoing work.
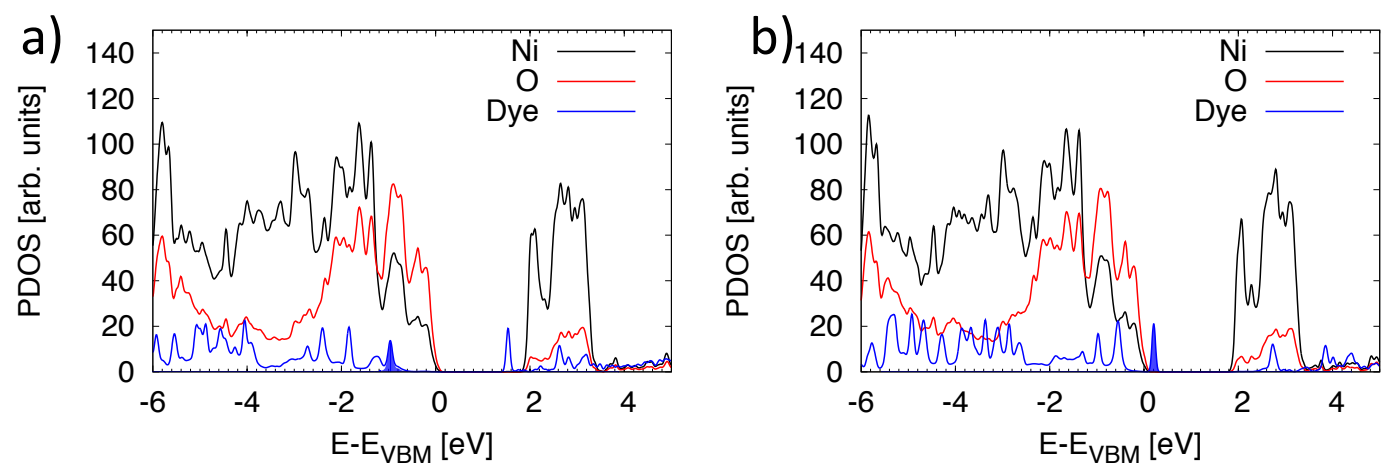

c)

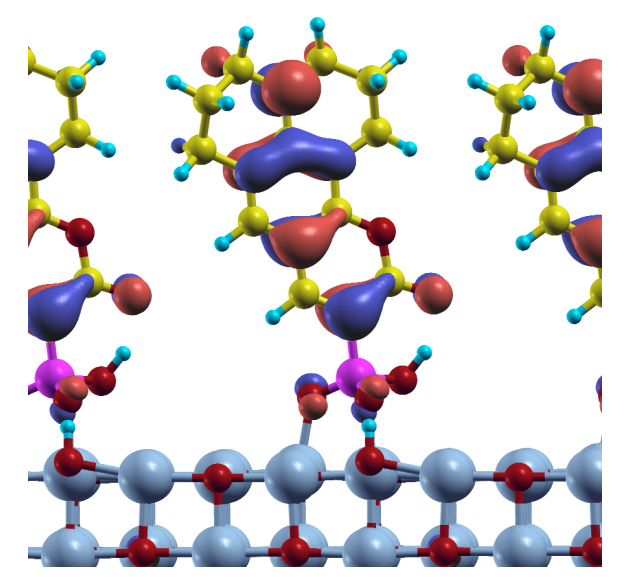

d)

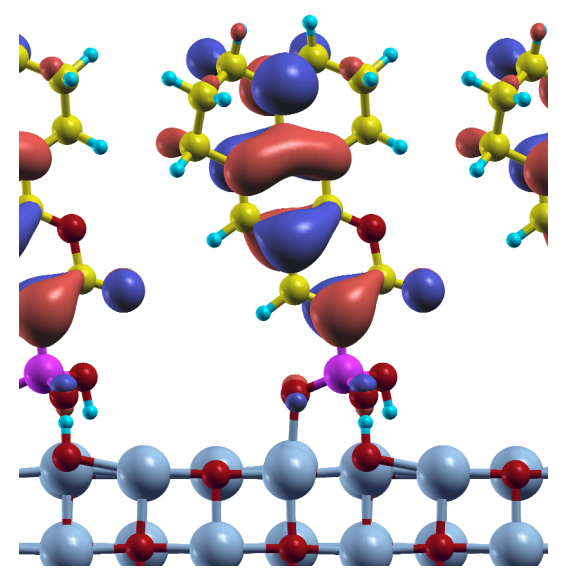

Figure 2: The $\mathrm{C} 343 / \mathrm{NiO}(100)$ system in vacuum in the bidentate anchoring geometry, evaluated using the PBE+U approach. We show the PDOS of: (a) the H-up geometry; (b) H-down geometry. The blue shaded areas represent the projections on the HOMO of the isolated dye. The isosurfaces of the dye's HOMO Kohn-Sham state are shown in panel (c) for the H-up geometry and panel (d) the H-down geometry. In both cases the dye's HOMO is fully localized on the dye, with no significant weight on the $\mathrm{NiO}$ slab.

Moving now to the adsorption of the dye on the surface, in line with previous work ${ }^{49}$ we find the bidentate binding to be the most stable anchoring mode of the $\mathrm{CH}_{3}-\mathrm{PO}(\mathrm{OH})_{2}$ group onto the $\mathrm{NiO}(100)$ surface (see SI for details) and we calculate an adsorption energy for the H-up and $\mathrm{H}$ - 
down C343 isomers adsorbed on our 6-layer surface slab of $-1.30 \mathrm{eV}$ and $-1.06 \mathrm{eV}$, respectively. In addition to the different adsorption energy, favoring the H-up configuration, the PDOS for the two systems in Fig. Figure 2 shows that there is also a large influence on the position of the molecular orbitals of the dye with respect to the NiO levels. In particular the HOMO of the dye lies $1.1 \mathrm{eV}$ below the VBM in the $\mathrm{H}$-up configuration, while it is $0.1 \mathrm{eV}$ above the VBM in the H-down geometry, the latter coinciding with the relative alignment predicted for the isolated components. An explanation for such a sizable shift can be found by looking at the electrostatic potential along the direction perpendicular to the $\mathrm{NiO}$ surface for the two geometries (Fig. S6 in SI). In the region of dye the two curves are shifted by $1.2 \mathrm{eV}$, in quantitative agreement with the overall shift of $1.2 \mathrm{eV}$ of the HOMO with respect to VBM induced by the change of the dye conformation. The different adsorption geometries result in different interfacial dipoles, which shift the electrostatic potential in the region of the dye relative to the potential of the $\mathrm{NiO}$ slab. Being essentially decoupled from the NiO slab (see plot of the corresponding Kohn-Sham state in Fig. Figure 2 and the sharp peak of the dye's HOMO), the HOMO of the dye experiences a rigid shift in energy with respect to the valence band of $\mathrm{NiO}$ as the positively charged $\mathrm{H}$ atom of the anchoring group moves from the H-down to the H-up configuration.

Summarizing, our gas phase " $0 \mathrm{~K}$ " picture of the C343-sensitized NiO interface sees the dye in the H-up conformation anchored stably in a bidentate mode to the metal oxide surface, characterized by an interfacial energy level lining up favorably for hole injection, with the dye's HOMO positioned about $1 \mathrm{eV}$ below the $\mathrm{NiO} \mathrm{VBM}$. Given the sensitivity of the dye/NiO level alignment to the change in interfacial dipoles, we expect that inclusion of water molecules and thermal fluctuations in the simulation will significantly alter the scenario discussed so far.

\section{$\mathrm{C343/NiO}$ in solution}

Turning now to our molecular dynamics simulations for the full system in water, in Fig. Figure 3 we first show the effect of the solvent on the level alignment evaluated at the initial snapshot (i.e. the snapshot extracted after the 3 ps equilibration). We compute the PDOS in three configurations: 

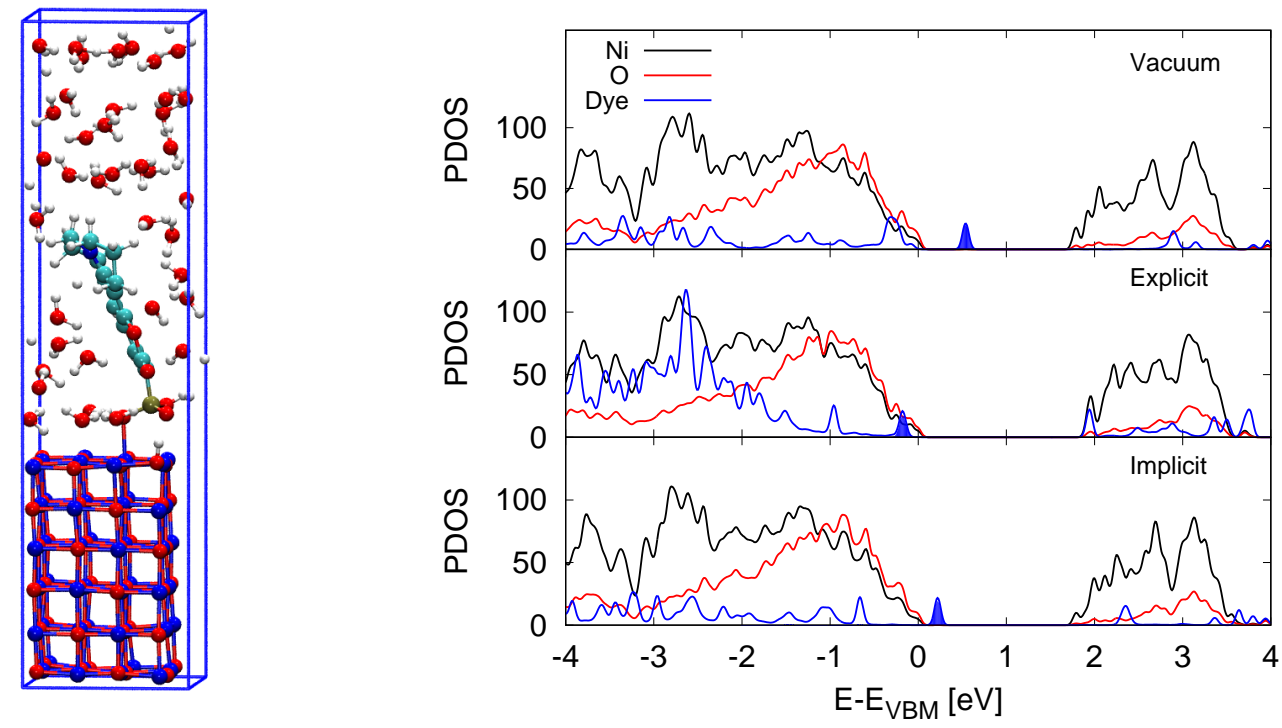

Figure 3: Effect of the liquid environment on the energy level alignment, evaluated on a snapshot at the very early stages of the production run. Left panel: Geometry of the full system. Right panel: PDOS evaluated in vacuum, in explicit water, and in implicit solvent. The area filled in solid color represents the projection of the Kohn-Sham states of the full system on the HOMO state of the isolated dye.

The full system with explicit water (labeled "Explicit" in Fig. Figure 3); The system where all the water molecules have been removed ("Vacuum"); The system where the solvent is modeled through an implicit solvent model ("Implicit"). In all cases the positions of the atoms of $\mathrm{NiO}$ and of the dye are identical. The color-filled peak in Fig. Figure 3 represents the projection of the Kohn-Sham states of the full system on the HOMO state of the isolated dye, enabling us to clearly identify the dye's HOMO within the full system. The effect of the solvent is to shift the levels of the dye towards lower energies, relative to the states of $\mathrm{NiO}$, from $0.7 \mathrm{eV}$ above the $\mathrm{VBM}$ in vacuum to $-0.2 \mathrm{eV}$ in explicit solvent. This effect is partly captured also using an implicit solvent model, even though the magnitude of the energy shift is significantly smaller than in the case of the explicit solvent, since the dye's HOMO is still $0.2 \mathrm{eV}$ above the NiO's VBM. Notice that the geometry in vacuum of this snapshot is quite different from the most stable H-up geometry considered earlier in vacuum, hence the large change in the level alignment.

To evaluate the influence of the exchange and correlation functional, we compare in Fig. S7 in SI the electronic structure of the system using the $\mathrm{PBE}+\mathrm{U}$ and $\mathrm{HSE}(\mathrm{X}=20 \%)$ functionals. Also in 
this case, the comparison has been made on the initial snapshot. While the effect of the inclusion of exact exchange is clearly to increase the $\mathrm{NiO}$ gap and to enhance the Ni character of the top of the valence band (as already discussed for bulk NiO, see Fig. S2 in SI), the position of the levels of the dye relative to $\mathrm{NiO}$ in not significantly affected, with the dye's HOMO being close to the top of the valence band of $\mathrm{NiO}$ in both cases.
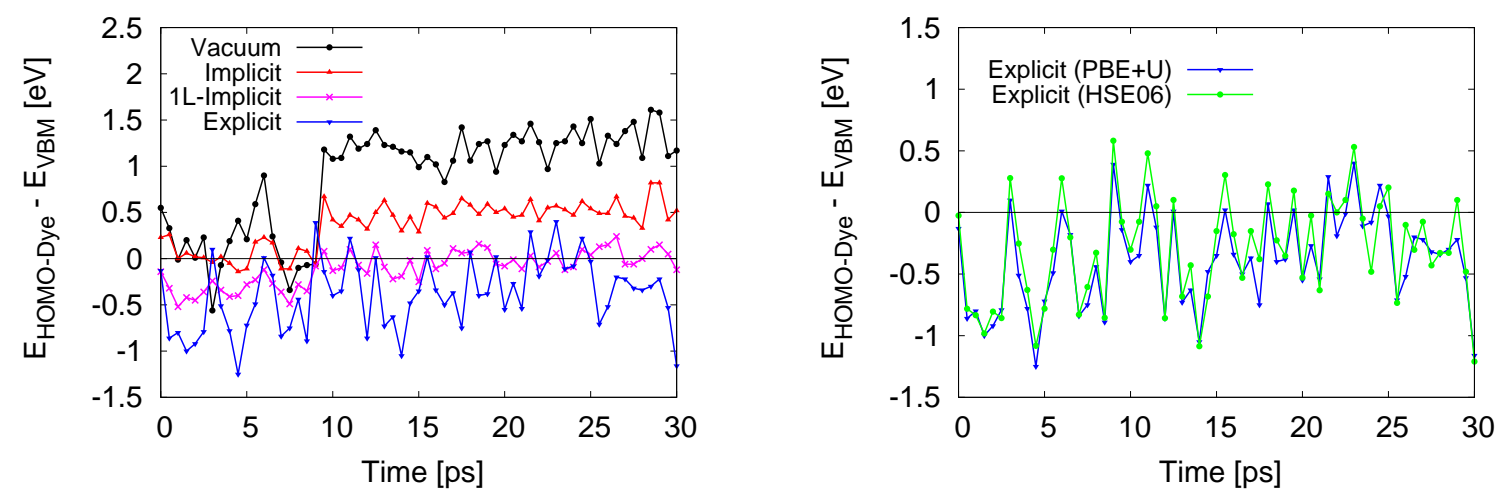

Figure 4: Position of the HOMO of the dye relative to the top of the valence band of $\mathrm{NiO}$ during the dynamics. Calculations in vacuum and in implicit solvent have been performed with the PBE+U method. The calculations in explicit water have been performed with both the PBE+U method and the $\operatorname{HSE}(X=20 \%)$ functional.

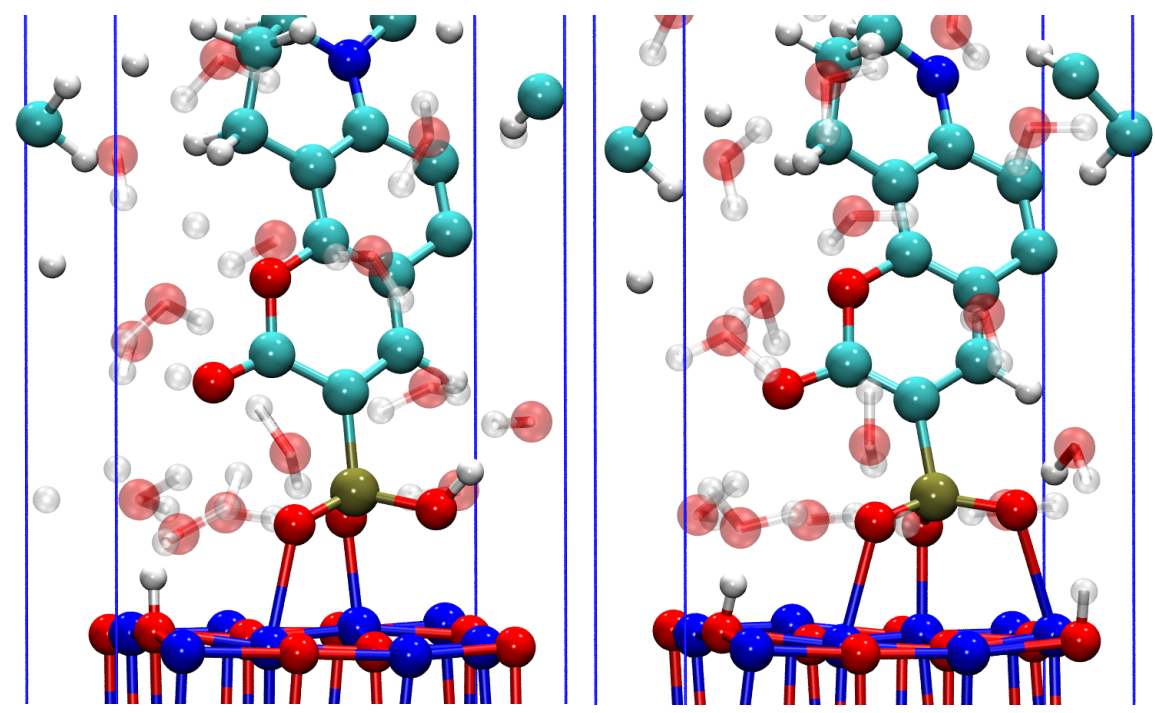

Figure 5: Change of anchoring geometry during the molecular dynamics in explicit water. Left: Bidentate geometry. Right: Tridentate geometry. 
In Fig. Figure 4, we show the position of HOMO of the dye relative to NiO's VBM across the whole $\sim 30$ ps dynamics. At around $9 \mathrm{ps}$, the initial bidentate anchoring geometry changes into a tridentate configuration, whereby a proton is transferred from the hydroxyl group of phosphate to the NiO surface (see Fig. Figure 5). In vacuum, however, the bidentate geometry is favored over the tridentate by $0.41 \mathrm{eV}$, while in the implicit solvent it is favored by $0.28 \mathrm{eV}$. To check whether this result is reproducible, we performed two further molecular dynamics simulations starting with different initial geometries of the dye and the solvent. In every case we observed a transition from a bidentate to a tridentate anchoring within a few ps. This geometrical change has a large effect on the energy level alignment evaluated in vacuum, since the dipole induced by the transfer of the proton is not screened by the solvent, resulting in an upward shift of the dye's HOMO relative to NiO's VBM. This effect is partially mitigated by the inclusion of an implicit solvent model, while in explicit water the alignment is weakly affected by such change of anchoring geometry. Further details of the solvent radial distribution functions and density are shown in Fig. S8 and S9 in SI.

Fig. Figure 4 shows that the fluctuations in the energy level alignment are larger in the case of explicit water, suggesting that the change in the instantaneous configuration of the water molecules surrounding the dye/NiO interface have a larger effect on the alignment than the change in the dye/NiO configuration. Considering time averages along the dynamics, results in implicit and explicit water differ substantially. Focusing on the portion of the dynamics where the dye anchors in a tridentate mode, we computed the average position of the dye's HOMO relative NiO's VBM, obtaining values of $1.22 \pm 0.17 \mathrm{eV}$ in vacuum, $0.51 \pm 0.12 \mathrm{eV}$ in implicit solvent and $-0.31 \pm$ $0.34 \mathrm{eV}$ in explicit solvent.

The large difference between the results obtained with implicit and explicit solvent models suggests that the water molecules at the dye/NiO interface do not behave like bulk water molecules, likely because of a strong interaction with the surface atoms, and are therefore not well described by the continuum solvent model. To test whether this is the case, we considered also a system in which the layer of water molecules bound to the surface is retained (there are four such molecules in our simulation cell) while the rest of the solvent is modeled through an implicit solvent model. 
As shown in Fig. Figure 4 the explicit inclusion of the first water layer improves the agreement with explicit solvent simulation, with an average position of the dye's HOMO relative NiO's VBM of $-0.01 \pm 0.11 \mathrm{eV}$.

We also find that the kind of functional used for the exchange and correlation potential does not have a large role in the level alignment. As we can see in Fig. Figure 4, the results obtained with explicit water molecules using the PBE+U approach and the HSE06 hybrid functional do not differ substantially. The average value obtained for HSE06 is $-0.22 \pm 0.38 \mathrm{eV}$, differing less than $0.1 \mathrm{eV}$ from the PBE+U value. This is in line with what we observed for the isolated systems, where both methods predicted the dye's HOMO to be slightly above (within $0.1 \mathrm{eV}$ ) NiO's VBM. The creation of the solvated interface therefore modifies the level alignment with respect to the isolated systems, moving the dye's HOMO down in energy with respect to NiO's VBM by $\sim 0.3$ eV. As stressed above, the approximations in our electronic structure methods likely destabilize the dye's HOMO. More accurate approaches could therefore give even more favorable level alignment for hole injection.

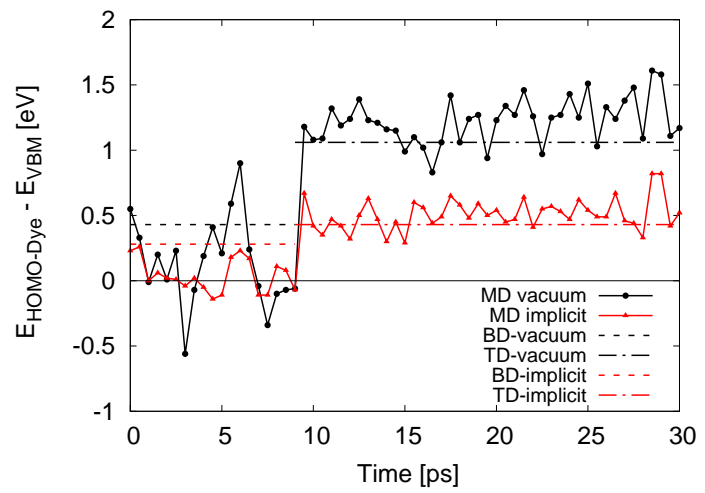

Figure 6: Energy level alignment of the dye's HOMO with respect to the maximum of NiO's valence band. MD refers to results obtained from snapshots extracted from the molecular dynamics simulations. BD and TD refer to bidentate and tridentate geometries, respectively.

In Fig. Figure 6 we compare the results obtained analyzing the snapshots extracted from the MD (in vacuum and in implicit solvent) with results obtained from geometry optimizations in vacuum and in an implicit solvent. The two sets of data agree quite well, showing that the energy 
level alignment obtained optimizing the structure (either in the bidentate or tridentate geometry) is consistent with the corresponding average value extracted from the MD. This, however, implies that for this system a static calculation cannot capture the effect of the solvent on the energy alignment across the interface. The favorable alignment we find with the simulation in explicit water is not reproduced with a static calculation where we optimize the geometry in implicit solvent. Finally, to test whether van der Waals contributions to the interatomic forces play any role in the energy level alignment, we performed an additional 10 ps molecular dynamics without such contributions. As shown in Fig. S10, the dye's HOMO continues to be favorably aligned with respect to NiO's VBM, and the energy difference between these two levels displays fluctuations comparable to those obtained with the van der Waals contributions, implying that these terms do not play a key role in the level alignment of this system.

\section{Conclusions}

In summary, we have shown that the energy level alignment $\mathrm{C} 343 / \mathrm{NiO}(100)$ interface displays a complex behavior. The alignment is largely determined by electrostatic effects induced by the anchoring geometry, the water layer bound to the surface and the solvation water. First principles molecular dynamics simulations, accounting explicitly for all these effects, correctly predict the HOMO of the dye to be below the valence band maximum of $\mathrm{NiO}$, therefore in a favorable position of hole injection. These predictions are strongly influenced by how the solvent is modeled, suggesting an explicit solvent model to be necessary to predict the correct alignment. We find, on the other hand, that the exchange and correlation functional does not have a dramatic effect on the alignment.

\section{Supporting Information Available}

In the Supporting Information we provide: the DOS of the isolated systems, position of the dye's HOMO relative to vacuum in various environments, binding energies of the dye, electrostatic po- 
tential alignment, geometries for the bi- and tri-dentate configurations in solution, analysis of radial distribution functions and water density, analysis of the effect of van der Waals forces on the energy level alignment.

\section{Acknowledgement}

D.R. acknowledges financial support from Agence Nationale de la Recherche under grant number ANR-15-CE29-0003-01. We acknowledge the CINECA award under the ISCRA initiative, for the availability of high performance computing resources and support. We also acknowledge HPC resources from GENCI-CCRT/CINES (Grants 2017-A0010810139 and x2017-085106) and the DECI resource ARCHER based in the United-Kingdom with support from PRACE.

\section{References}

(1) Grätzel, M. J. Photochem. Photobiol. C: Photochem. Rev. 2003, 4, 145-153.

(2) Grätzel, M. Acc. Chem. Res. 2009, 42, 1788-1798.

(3) Hagfeldt, A.; Boschloo, G.; Sun, L.; Kloo, L.; Pettersson, H. Chem. Rev. 2010, 110, 65956663.

(4) Alibabaei, L.; Brennaman, M. K.; Norris, M. R.; Kalanyan, B.; Song, W.; Losego, M. D.; Concepcion, J. J.; Binstead, R. A.; Parsons, G. N.; Meyer, T. J. Proc. Natl. Acad. Sci. 2013, 110, 20008-20013.

(5) Walter, M. G.; Warren, E. L.; McKone, J. R.; Boettcher, S. W.; Mi, Q.; Santori, E. A.; Lewis, N. S. Chem. Rev. 2010, 110, 6446-6473.

(6) Youngblood, W. J.; Lee, S.-H. A.; Maeda, K.; Mallouk, T. E. Acc. Chem. Res. 2009, 42, 1966-1973.

(7) Yu, Z.; Li, F.; Sun, L. Energy Environ. Sci. 2015, 8, 760-775. 
(8) O’Regan, B.; Grätzel, M. Nature 1991, 353, 737-740.

(9) He, J.; Lindström, H.; Hagfeldt, A.; Lindquist, S.-E. J.Phys. Chem. B 1999, 103, 8940-8943.

(10) Borgström, M.; Blart, E.; Boschloo, G.; Mukhtar, E.; Hagfeldt, A.; Hammarström, L.; Odobel, F. J. Phys. Chem. B 2005, 109, 22928-22934.

(11) Odobel, F.; Pellegrin, Y.; Gibson, E. A.; Hagfeldt, A.; Smeigh, A. L.; Hammarström, L. Coord. Chem. Rev. 2012, 256, 2414 - 2423.

(12) Brennaman, M. K.; Dillon, R. J.; Alibabaei, L.; Gish, M. K.; Dares, C. J.; Ashford, D. L.; House, R. L.; Meyer, G. J.; Papanikolas, J. M.; Meyer, T. J. J. Am. Chem. Soc. 2016, 138, $13085-13102$.

(13) He, J.; Lindström, H.; Hagfeldt, A.; Lindquist, S.-E. Sol. Energy Mater Sol. Cells 2000, 62, $265-273$.

(14) Odobel, F.; Farre, Y.; Raissi, M.; Fihey, A.; Pellegrin, Y.; Blart, E.; Jacquemin, D. ChemSusChem 2017, 10, 2618-2625.

(15) Ji, Z.; He, M.; Huang, Z.; Ozkan, U.; Wu, Y. J. Am. Chem. Soc. 2013, 135, 11696-11699.

(16) Sherman, B. D.; Sheridan, M. V.; Wee, K.-R.; Marquard, S. L.; Wang, D.; Alibabaei, L.; Ashford, D. L.; Meyer, T. J. J. Am. Chem. Soc. 2016, 138, 16745-16753.

(17) Kaeffer, N.; Massin, J.; Lebrun, C.; Renault, O.; Chavarot-Kerlidou, M.; Artero, V. J. Am. Chem. Soc. 2016, 138, 12308-12311.

(18) Li, L.; Duan, L.; Wen, F.; Li, C.; Wang, M.; Hagfeldt, A.; Sun, L. Chem. Commun. 2012, 48, 988-990.

(19) Gardner, J. M.; Beyler, M.; Karnahl, M.; Tschierlei, S.; Ott, S.; Hammarström, L. J. Am. Chem. Soc. 2012, 134, 19322-19325. 
(20) Antila, L. J.; Ghamgosar, P.; Maji, S.; Tian, H.; Ott, S.; Hammarström, L. ACS En. Lett. 2016, $1,1106-1111$.

(21) Powar, S.; Daeneke, T.; Ma, M. T.; Fu, D.; Duffy, N. W.; Götz, G.; Weidelener, M.; Mishra, A.; Bäuerle, P.; Spiccia, L.; Bach, U. Ang. Chem. Int. Ed. 2013, 52, 602-605.

(22) Perera, I. R.; Daeneke, T.; Makuta, S.; Yu, Z.; Tachibana, Y.; Mishra, A.; Bäuerle, P.; Ohlin, C. A.; Bach, U.; Spiccia, L. Ang. Chem. Int. Ed. 2015, 54, 3758-3762.

(23) Gibson, E. A.; Smeigh, A. L.; Le Pleux, L.; Hammarström, L.; Odobel, F.; Boschloo, G.; Hagfeldt, A. J. Phys. Chem. C 2011, 115, 9772-9779.

(24) Qin, P.; Zhu, H.; Edvinsson, T.; Boschloo, G.; Hagfeldt, A.; Sun, L. J. Am. Chem. Soc. 2008, $130,8570-8571$.

(25) Gennari, M.; Légalité, F.; Zhang, L.; Pellegrin, Y.; Blart, E.; Fortage, J.; Brown, A. M.; Deronzier, A.; Collomb, M.-N.; Boujtita, M.; Jacquemin, D.; Hammarström, L.; Odobel, F. J. Phys. Chem. Lett. 2014, 5, 2254-2258.

(26) Ji, Z.; Natu, G.; Huang, Z.; Wu, Y. Energy Environ. Sci. 2011, 4, 2818-2821.

(27) Wood, C. J.; McGregor, C. A.; Gibson, E. A. ChemElectroChem 2016, 3, 1827-1836.

(28) D’Amario, L.; Boschloo, G.; Hagfeldt, A.; Hammarström, L. J. Phys. Chem. C 2014, 118, 19556-19564.

(29) Biju, V.; Khadar, M. A. Mater. Res. Bull. 2001, 36, 21-33.

(30) Morandeira, A.; Boschloo, G.; Hagfeldt, A.; Hammarström, L. J. Phys. Chem. B 2005, 109, 19403-19410.

(31) Warnan, J.; Gardner, J.; Le Pleux, L.; Petersson, J.; Pellegrin, Y.; Blart, E.; Hammarström, L.; Odobel, F. J. Phys. Chem. C 2014, 118, 103-113. 
(32) Zhang, L.; Favereau, L.; Farre, Y.; Mijangos, E.; Pellegrin, Y.; Blart, E.; Odobel, F.; Hammarström, L. Phys. Chem. Chem. Phys. 2016, 18, 18515-18527.

(33) Smeigh, A. L.; Pleux, L. L.; Fortage, J.; Pellegrin, Y.; Blart, E.; Odobel, F.; Hammarström, L. Chem. Commun. 2012, 48, 678-680.

(34) Brown, A. M.; Antila, L. J.; Mirmohades, M.; Pullen, S.; Ott, S.; Hammarström, L. J. Am. Chem. Soc. 2016, 138, 8060-8063.

(35) Bonomo, M.; Dini, D.; Marrani, A. G. Langmuir 2016, 32, 11540-11550.

(36) Odobel, F.; Le Pleux, L.; Pellegrin, Y.; Blart, E. Acc. Chem. Res. 2010, 43, 1063-1071.

(37) Boschloo, G.; Hagfeldt, A. Acc. Chem. Res. 2009, 42, 1819-1826.

(38) Persson, P.; Bergström, R.; Lunell, S. J. Phys. Chem. B 2000, 104, 10348-10351.

(39) De Angelis, F.; Fantacci, S.; Selloni, A.; Nazeeruddin, M. K.; Grätzel, M. J. Am. Chem. Soc. 2007, 129, 14156-14157.

(40) Martsinovich, N.; Troisi, A. Energy Environ. Sci. 2011, 4, 4473-4495.

(41) Agrawal, S.; Leijtens, T.; Ronca, E.; Pastore, M.; Snaith, H.; De Angelis, F. J. Mater. Chem. A 2013, 1, 14675-14685.

(42) Le Bahers, T.; Pauporté, T.; Lainé, P. P.; Labat, F.; Adamo, C.; Ciofini, I. J. Phys. Chem. Lett. 2013, 4, 1044-1050.

(43) Pastore, M.; De Angelis, F. In Modeling materials and processes in dye-sensitized solar cells: Understanding the mechanism, improving the efficiency; Beljonne, D., Cornil, J., Eds.; Springer Berlin Heidelberg: Berlin, Heidelberg, 2014; pp 151-236.

(44) Mosconi, E.; Selloni, A.; De Angelis, F. J. Phys. Chem. C 2012, 116, 5932-5940. 
(45) Monti, A.; Negre, C. F. A.; Batista, V. S.; Rego, L. G. C.; de Groot, H. J. M.; Buda, F. J. Phys. Chem. Lett. 2015, 6, 2393-2398.

(46) Pastore, M. Computation 2017, 5, 5.

(47) De Angelis, F. Acc. Chem. Res. 2014, 47, 3349-3360.

(48) Mosconi, E.; Etienne, T.; De Angelis, F. In First-principles modeling of organohalide thin films and interfaces; Park, N.-G., Grätzel, M., Miyasaka, T., Eds.; Springer International Publishing: Cham, 2016; pp 19-52.

(49) Munoz-Garcia, A. B.; Pavone, M. Phys. Chem. Chem. Phys. 2015, 12238-12246.

(50) Kontkanen, O. V.; Niskanen, M.; Hukka, T. I.; Rantala, T. T. Phys. Chem. Chem. Phys. 2016, $18,14382-14389$.

(51) Wykes, M.; Odobel, F.; Adamo, C.; Ciofini, I.; Labat, F. J. Mol. Model. 2016, 22, 289.

(52) Perdew, J. P.; Burke, K.; Ernzerhof, M. Phys. Rev. Lett. 1996, 77, 3865.

(53) Cococcioni, M.; de Gironcoli, S. Phys. Rev. B 2005, 71, 035105.

(54) Vanderbilt, D. Phys. Rev. B 1990, 41, 7892.

(55) Grimme, S. J. Comp. Chem. 2006, 27, 1787-1799.

(56) Giannozzi, P. et al. J. Phys. Cond. Matt. 2009, 21, 395502.

(57) Ravikumar, A.; Baby, A.; Lin, H.; Brivio, G. P.; Fratesi, G. Sci. Rep. 2016, 6, 24603.

(58) Bussi, G.; Donadio, D.; Parrinello, M. J. Chem. Phys. 2007, 126, 014101.

(59) Krukau, A. V.; Vydrov, O. A.; Izmaylov, A. F.; Scuseria, G. E. J. Chem. Phys. 2006, 125, 224106.

(60) Powell, J.; Spicer, W. E. Phys. Rev. B 1970, 2, 2182. 
(61) Hüfner, S.; Steiner, P.; Sander, I.; Reinert, F.; Schmitt, H. Zeitschrift für Physik B Cond. Matt. 1992, 86, 207-215.

(62) Hüfner, S. Advances in Physics 1994, 43, 183-356.

(63) Kurmaev, E. Z.; Wilks, R. G.; Moewes, A.; Finkelstein, L. D.; Shamin, S. N.; Kuneš, J. Phys. Rev. B 2008, 77, 165127.

(64) Faleev, S. V.; van Schilfgaarde, M.; Kotani, T. Phys. Rev. Lett. 2004, 93, 126406.

(65) Rödl, C.; Fuchs, F.; Furthmüller, J.; Bechstedt, F. Phys. Rev. B 2009, 79, 235114.

(66) Jiang, H.; Gomez-Abal, R. I.; Rinke, P.; Scheffler, M. Phys. Rev. B 2010, 82, 045108.

(67) Li, J.-L.; Rignanese, G.-M.; Louie, S. G. Phys. Rev. B 2005, 71, 193102.

(68) Dudarev, S. L.; Botton, G. A.; Savrasov, S. Y.; Humphreys, C. J.; Sutton, A. P. Phys. Rev. B 1998, 57, 1505-1509.

(69) Bengone, O.; Alouani, M.; Blöchl, P.; Hugel, J. Phys. Rev. B 2000, 62, 16392-16401.

(70) Jiang, H.; Gomez-Abal, R. I.; Rinke, P.; Scheffler, M. Phys. Rev. B 2010, 82, 045108.

(71) de P. R. Moreira, I.; Illas, F.; Martin, R. L. Phys. Rev. B 2002, 65, 155102.

(72) Morandeira, A.; Boschloo, G.; Hagfeldt, A.; Hammarström, L. J. Phys. Chem. C 2008, 112, 9530-9537.

(73) Mori, S.; Fukuda, S.; Sumikura, S.; Takeda, Y.; Tamaki, Y.; Suzuki, E.; Abe, T. J. Phys. Chem. C 2008, 112, 16134-16139.

(74) Nattestad, A.; Ferguson, M.; Kerr, R.; Cheng, Y.-B.; Bach, U. Nanotech. 2008, 19, 295304.

(75) Hod, I.; Tachan, Z.; Shalom, M.; Zaban, A. Phys. Chem. Chem. Phys. 2013, 15, 6339-6343.

(76) Pastore, M.; De Angelis, F. J. Am. Chem. Soc. 2015, 137, 5798-5809. 
(77) Brown, D. G.; Schauer, P. A.; Borau-Garcia, J.; Fancy, B. R.; Berlinguette, C. P. J. Am. Chem. Soc. 2013, 135, 1692-1695.

(78) De Angelis, F.; Fantacci, S.; Gebauer, R. J. Chem. Phys. Lett. 2011, 2, 813-817.

(79) Galliano, S.; Bella, F.; Gerbaldi, C.; Falco, M.; Viscardi, G.; Grätzel, M.; Barolo, C. Energy Technology 2017, 5, 300-311.

(80) Wu, W.; Cao, Z.; Zhao, Y. J. Chem. Phys. 2012, 136, 114305.

(81) Cave, R. J.; Castner, E. W. J. Phys. Chem. A 2002, 106, 12117-12123.

(82) Szuber, J. J. Electron Spectr. Rel. Phen. 1984, 34, 337-347.

(83) Tench, D. M.; Yeager, E. Electrochem. Sci. Tech. 1973, 120, 164-171.

(84) Madjid, A. H.; Martinez, J. M. Phys. Rev. Lett. 1972, 28, 1313-1315.

(85) Irwin, M. D.; Buchholz, D. B.; Hains, A. W.; Chang, R. P. H.; Marks, T. J. Proc. Natl. Acad. Sci. U.S.A. 2008, 105, 2783-2787.

(86) Nattestad, A.; Ferguson, M.; Kerr, R.; Cheng, Y.-B.; Bach, U. Nanotech. 2008, 19, 295304.

(87) Onida, G.; Reining, L.; Rubio, A. Rev. Mod. Phys. 2002, 74, 601-659.

(88) Ping, Y.; Rocca, D.; Galli, G. Chem. Soc. Rev. 2013, 42, 2437-2469. 